\title{
Metastatic papillary thyroid cancers with malignant pleural effusion aggravated during thyroid hormone withdrawal for radioiodine therapy
}

Ji Hye Seo, Ji Hye Je, Hyun Jung Lee, Young Ju Na, II Woo Jeong, Jee Hyun An, Sin Gon Kim, Dong Seop Choi, Nam Hoon Kim

Department of Internal Medicine, Korea University College of Medicine, Seoul, Korea

L-thyroxine (LT4) withdrawal prior to radioactive iodine (RAI) ablation therapy is a commonly used method for successful treatment of patients with papillary thyroid cancer (PTC). However, a prolonged period of hypothyroidism induced by LT4 withdrawal is sometimes associated with impaired quality of life and cardiopulmonary dysfunction in PTC patients. Furthermore, LT4 withdrawal may have a trophic effect on residual cancer by means of increased thyrotropin. We report on 2 cases of metastatic PTC patients with malignant pleural effusion (MPE) whose disease showed rapid worsening after LT4 withdrawal and RAI therapy. The first case is a 65-year-old woman who had PTC with multiple distant metastases and MPE. During LT4 withdrawal for RAl therapy, MPE showed rapid worsening, and the patient required repetitive therapeutic thoracentesis. The second case is a 49-year-old woman with PTC who underwent 3 additional operations for cancer recurrence in the neck lymph nodes and 6 times of RAl treatments. While preparing for the $7^{\text {th }}$ RAI treatment by withdrawing LT4, she developed MPE which became progressively aggravated after RAI therapy. Both patients experienced increased pleural effusion during the LT4 withdrawal period and a rise in the thyroglobulin level was observed after RAI therapy. MPE was not controlled with therapeutic thoracentesis and pleurodesis. Eventually, both patients died of rapid disease progression after RAl therapy. In summary, LT4 withdrawal may have an adverse effect on metastatic PTC patients, particularly those with MPE.

Keywords: Thyroid neoplasms; Thyroid hormones; Malignant pleural effusion

\section{INTRODUCTION}

Papillary thyroid cancer (PTC) is generally known to have a good prognosis after definitive primary treatment with total or near-total thyroidectomy, radioactive iodine (RAI) ablation therapy, and subsequent suppression of endogenous thyroidstimulating hormone (TSH) with thyroid hormones. However, approximately 5\%-20\% of patients experience local recurrences or distant metastases that may require further active

Received: July 16, 2014, Revised: September 19, 2014 , Accepted: September 22, 2014

Corresponding Author: Nam Hoon Kim, Division of Endocrinology and Metabolism, Department of Internal Medicine, Korea University Anam Hospital, Korea University College of Medicine, 73 Inchon-ro, Seongbuk-gu, Seoul 02841, Korea

Tel: +82-2-920-5890, Fax: +82-2-953-9355

E-mail: pourlife@naver.com treatments [1]. Distant metastases, mainly into the lung or bones, account for nearly $5 \%$ of patients, and relapsing as pleural effusion is rare [2]. A large case series over a period of 47 years reported that only $0.6 \%$ of patients with PTC had metastatic pleural effusion [3].

Most patients with metastatic PTC undergo RAI therapy. Before RAI therapy, withdrawal of L-thyroxine (LT4) for several weeks is carried out, which results in a prolonged period of hypothyroidism. Although temporary thyroid hormone withdrawal is generally well tolerated in most patients, it may lower quality of life [4], and it is sometimes associated with undesirable cardiovascular effects [5-8]. In addition, the prolonged rise in serum TSH may promote tumor progression in metastatic PTC patients [9].

Here, we describe 2 patients with metastatic papillary thyroid cancer with lung metastasis and malignant pleural effusion (MPE), whose medical condition became rapidly aggravated during thyroid hormone withdrawal for radioiodine therapy. 


\section{CASES}

\section{Case 1}

In 2008, a 65-year-old woman was referred to our hospital for evaluation of a palpable neck mass, and PTC was suggested by fine-needle aspiration cytology. The patient underwent total thyroidectomy with central lymph node dissection, and histology demonstrated papillary carcinoma (right lobe, $2.2 \mathrm{~cm}$ ) with minimal extrathyroidal extension and metastasis in 1 out of 6 regional lymph nodes. After the operation, I-131 was administered at a dose of $150 \mathrm{mCi}$. Post-ablation whole body scan (WBS) revealed a residual uptake only in the thyroid bed. Diagnostic WBS obtained 1 year after surgery did not reveal any remnant tissue or metastasis, and serum thyroglobulin (Tg) level during LT4 withdrawal (T4off $\mathrm{Tg}$ ) was $0.1 \mathrm{ng} / \mathrm{mL}$ with thyroglobulin antibody (Tg-Ab) level at $8.6 \mathrm{IU} / \mathrm{mL}$. Four years after, the operation, serum thyroglobulin level during thyroxine administration (T4-on $\mathrm{Tg}$ ) increased to $7.77 \mathrm{ng} / \mathrm{mL}$ (Tg-Ab $48.4 \mathrm{IU} / \mathrm{mL}$ ), but neck ultrasonography did not show definite local recurrence or nodal metastasis. After 6 months, T4-on Tg level showed a rapid increase up to $64.02 \mathrm{ng} / \mathrm{mL}$ (Tg-Ab $26.4 \mathrm{IU} / \mathrm{mL}$ ).

Shortly after this (examination December 2012), the patient was admitted to the emergency room due to dyspnea. A chest radiograph showed a large amount of right pleural effusion (Fig. 1A), and malignant cells (papillary carcinoma) were detected in the pleural fluid by thoracentesis. Subsequent chest computed tomography (CT) and positron emission tomography (PET) CT scans revealed the presence of metastases in the lung, pleura and bone (left second rib) as well as pleural effusion (Fig. 1B-D). The patient had been hospitalized for 16 days, and she underwent therapeutic thoracentesis once during hospitalization. For the second RAI ablation, thyroxine was discontinued, and tri-iodothyronine (60 $\mu \mathrm{g} /$ day) was administered and discontinued after 2 weeks. Pleural effusion increased more rapidly during the 2 weeks of thyroid hormone withdrawal (Fig. 1E, 1F), and therapeutic thoracentesis was performed more frequently (a total of 4 times in 2 weeks).

After 2 weeks of tri-iodothyronine withdrawal, the TSH level increased to $>100 \mu \mathrm{IU} / \mathrm{L}$, and the T4-off Tg level increased up to $146.97 \mathrm{ng} / \mathrm{mL}$ (Tg-Ab $29.5 \mathrm{IU} / \mathrm{mL})$. I-131 (200 mCi) was administered, and the subsequent WBS showed iodine up- take in the right axilla and right hemithorax. Even after re-administration of thyroxine supplements and repetitive thoracentesis, the large amount of pleural effusion was not resolved. Consequently, a right thoracostomy was performed, and pleurodesis was done 4 times. Pleurodesis attenuated the increase in pleural effusion for approximately 2 months, and level of TSH decreased to $0.85 \mu \mathrm{IU} / \mathrm{L}$. However, T4-on Tg level increased progressively even after RAI therapy (T4-on Tg; 239 $\mathrm{ng} / \mathrm{mL}$ at 2 months, $810 \mathrm{ng} / \mathrm{mL}$ at 5 months, and 1,282 ng/mL at 7 months after RAI therapy). We had planned additional RAI therapy, however, dyspnea of the patient was aggravated and her general condition showed considerable deterioration. Finally, she died 9 months after her second RAI therapy.

\section{Case 2}

A 49-year-old woman was diagnosed with PTC in 2005. She underwent total thyroidectomy with neck node dissec-
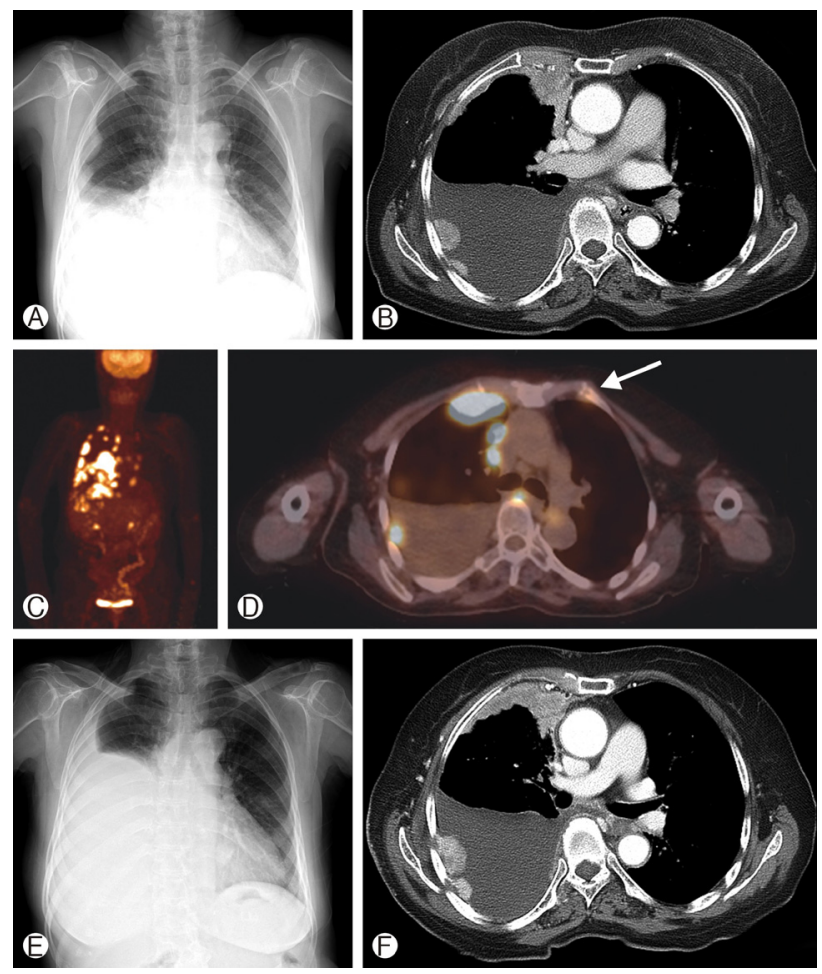

Fig. 1. (A) Chest radiograph shows a large amount of right pleural effusion. Chest computed tomography (CT) (B) and positron-emission tomography CT (C, D) scans show metastases in the lungs, pleura and left second rib (arrow), as well as pleural effusion. A chest radiograph (E) and chest CT scans $(\mathrm{F})$ obtained 2 weeks after withdrawal of L-thyroxine administration show increased right pleural effusion and pleural metastasis. 
tion, and histology demonstrated papillary carcinoma with $1.5 \mathrm{~cm}$ in the right lobe, $1.7 \mathrm{~cm}$ in the left lobe, with an extension to the perithyroidal soft tissue and metastases in 42 out of 111 regional lymph nodes. Subsequently, she underwent RAI therapy (150 mCi of I-131). The post-ablation WBS showed residual thyroid uptake. T4-off $\mathrm{Tg}$ and $\mathrm{Tg}$-Ab before RAI therapy was $1,437 \mathrm{ng} / \mathrm{mL}$ and $20 \mathrm{IU} / \mathrm{mL}$. After the first RAI therapy, she had suffered from repetitive recurrences of PTC at the cervical lymph nodes. During 4 years following the initial operation, she underwent 3 additional operations, 4 additional rounds of RAI therapy (a total of $600 \mathrm{mCi}$ of I-131), and 2 sessions of external radiation therapy. A PET-CT scan obtained 5 years after the initial operation (March 2010) demonstrated multiple metastatic nodules in both lungs. Thus, she received a 6th round of RAI therapy $(200 \mathrm{mCi}$ of I-131, TSH >100 $\mu \mathrm{IU} / \mathrm{L}$, T4-off Tg 103.14 ng/mL, T4-off Tg-Ab 23.9 $\mathrm{IU} / \mathrm{mL}$ ). However, the T4-on Tg level increased progressively as follows: $83 \mathrm{ng} / \mathrm{mL}$ at 20 months, $116.69 \mathrm{ng} / \mathrm{mL}$ at 26 months, $117.28 \mathrm{ng} / \mathrm{mL}$ at 33 months, and $137.46 \mathrm{ng} / \mathrm{mL}$ at 36 months after the 6th round of RAI therapy.

A PET-CT scan taken 33 months after the 6th RAI therapy (February 2013) showed aggravated metastatic lung lesions. Therefore, we planned an additional RAI therapy. Thyroxine was replaced with tri-iodothyronine, which was discontinued 2 weeks prior to RAI therapy. At the admission for the 7th RAI therapy, a chest radiograph showed left pleural effusion, which had not been previously observed (Fig. 2A). T4-off Tg level had increased to $304 \mathrm{ng} / \mathrm{mL}$. I-131 (200 mCi) was administered, and a subsequent WBS showed nodular iodine uptake in the right chest. Thyroxine (200 $\mu \mathrm{g} /$ day) was re-administered, and the patient was discharged. Fourteen days after RAI therapy, she was admitted to the emergency room for whole body myalgia. Laboratory data showed elevated TSH and Tg-Ag levels of $52.56 \mathrm{mIU} / \mathrm{L}$ and $396.9 \mathrm{ng} / \mathrm{mL}$, respectively. A chest radiograph showed an increase in pleural effusion, and a chest CT scan showed an increase in size and number of metastatic nodules in both lungs and pleura, as well as a large amount of left pleural effusion (Fig. 2B, 2C). Left thoracostomy was performed, and pleurodesis was performed 3 times. A month after RAI therapy, she complained of dyspnea. A chest radiograph and chest CT scan revealed progression of pleuropulmonary metastases (Fig. 2D, 2E). She and her family opted for conservative care rather than aggressive treatment. Eventually, she died of respiratory
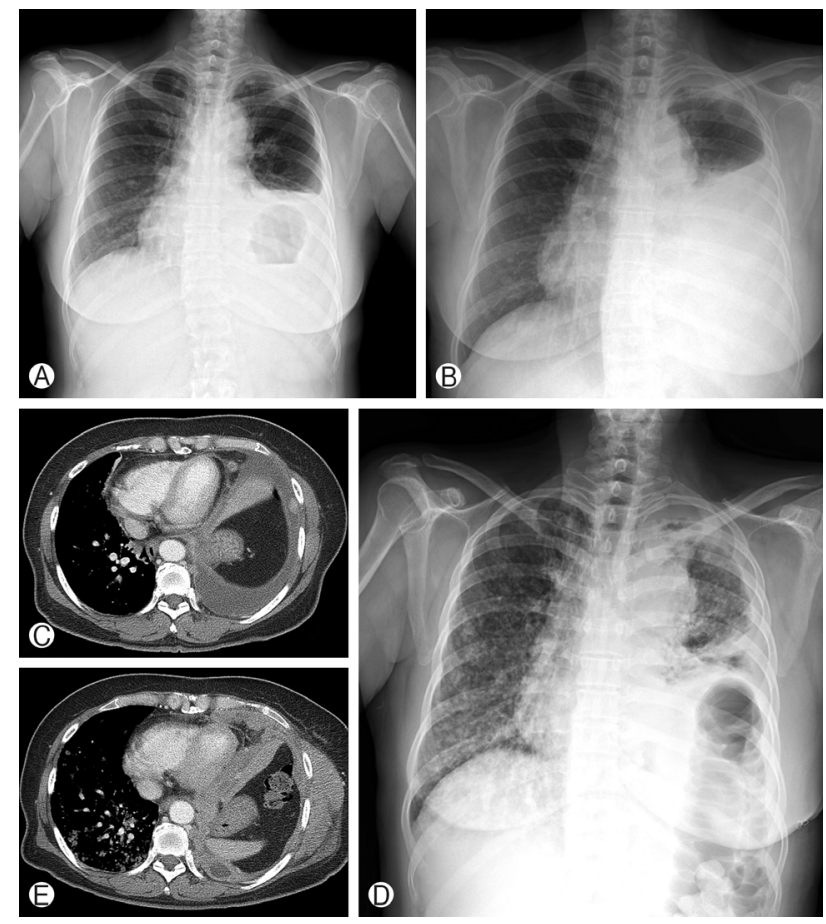

Fig. 2. (A) A chest radiograph obtained 2 weeks after withdrawal of thyroid hormone administration shows left pleural effusion. (B) A chest radiograph obtained 2 weeks after radioactive iodine therapy shows increased left pleural effusion. (C) A chest computed tomography (CT) scan obtained 2 weeks after radioactive iodine therapy shows increased metastatic nodules in both lungs and the pleura as well as a large amount of left pleural effusion. A chest radiograph (D) and chest CT scan (E) obtained 34 days after radioactive iodine therapy show progression of pleuropulmonary metastases.

failure due to pulmonary metastases in August 2013.

\section{DISCUSSION}

In this report, we presented 2 cases of metastatic PTC with extensive lung metastasis and MPE whose disease showed rapid worsening after LT4 withdrawal and RAI therapy. Both cases involved high-risk patients who had extrathyroidal invasion and lymph node metastasis at the time of diagnosis and subsequently developed distant metastasis. They had a large amount of pleural effusion and died owing to rapid disease progression after RAI therapy. The rapid increase in pleural effusion and a rise in the T4-on Tg level after RAI therapy in our cases suggested that LT4 withdrawal may adversely affects patients with metastatic PTC, especially those with MPE.

The pleural effusion increased rapidly during LT4 withdrawal in these 2 cases. This might result from tumor pro- 
gression, but it could also be from various harmful effects of LT4 withdrawal. LT4 withdrawal results in a prolonged period of hypothyroidism which may induce or aggravate pleural effusion. Hataya et al., who reported on a case of subclinical hypothyroidism who developed marked pleural effusion and peripheral edema which were ameliorated after thyroid hormone replacement, suggested that elevated vascular endothelial growth factor in hypothyroid patients might contribute to extravascular volume overload [10]. Parving et al. [11] reported that increased extravasations of plasma proteins and lack of a compensatory increase in lymph flow were observed in hypothyroid state. In addition, both overt and subclinical hypothyroidism are associated with undesirable cardiovascular effects including systolic and diastolic dysfunction both at rest and during effort, and increased peripheral vascular resistance [5,6]. Some studies have suggested that short term hypothyroidism induced by LT4 withdrawal can also lead to a deterioration of cardiac function, especially in terms of diastolic dysfunction [7,8]. Therefore, hypothyroidism itself and impaired cardiac function due to hypothyroidism may explain the worsening of pleural effusion in our cases.

Furthermore, a potential risk of thyroid hormone withdrawal is a prolonged exposure to increased level of TSH, which could stimulate cancer progression [9]. Kang et al. previously reported 4 high-risk PTC patients with metastasis who showed rapid deterioration after RAI therapy. Among those 4 patients, development of pleural effusion occurred after RAI therapy in 2 patients. They suggested that an increased TSH after LT4 withdrawal leads to rapid disease progression via a trophic effect on residual cancer [12]. In our cases, a rise in the Tg level after RAI therapy was observed, which could be an indirect evidence of tumor progression.

Currently, recombinant human TSH (rhTSH) is another method of preparing for RAI therapy. Previous studies have already shown that rhTSH is as effective as LT4 withdrawal in both low low-risk and high-risk patients including those with distant metastasis [13-15]. Despite its high cost, rhTSH has obvious advantages over LT4 withdrawal in terms of its ability to minimize the deterioration of cardiovascular function by reducing the period of hypothyroidism and tumor progression by shortening the period of elevated TSH. Therefore, rhTSH could be considered as an overriding method in preparing for RAI therapy in metastatic PTC patients, particularly those with MPE.
In summary, these 2 cases indicated that LT4 withdrawal prior to radioiodine therapy may have an adverse effect on MPE in patients with PTC.

\section{CONFLICT OF INTEREST}

The authors have no conflict of interest.

\section{REFERENCES}

1. Cho BY, Choi HS, Park YJ, Lim JA, Ahn HY, Lee EK, et al. Changes in the clinicopathological characteristics and outcomes of thyroid cancer in Korea over the past four decades. Thyroid 2013;23:797-804.

2. Borschitz T, Eichhorn W, Fottner C, Hansen T, Schad A, Schadmand-Fischer S, et al. Diagnosis and treatment of pancreatic metastases of a papillary thyroid carcinoma. Thyroid 2010;20:93-8.

3. Vassilopoulou-Sellin R, Sneige N. Pleural effusion in patients with differentiated papillary thyroid cancer. South Med J 1994;87:1111-6.

4. Dow KH, Ferrell BR, Anello C. Quality-of-life changes in patients with thyroid cancer after withdrawal of thyroid hormone therapy. Thyroid 1997;7:613-9.

5. Klein I, Ojamaa K. Thyroid hormone and the cardiovascular system. N Engl J Med 2001;344:501-9.

6. Biondi B, Klein I. Hypothyroidism as a risk factor for cardiovascular disease. Endocrine 2004;24:1-13.

7. Botella-Carretero JI, Gómez-Bueno M, Barrios V, Caballero C, García-Robles R, Sancho J, et al. Chronic thyrotropinsuppressive therapy with levothyroxine and short-term overt hypothyroidism after thyroxine withdrawal are associated with undesirable cardiovascular effects in patients with differentiated thyroid carcinoma. Endocr Relat Cancer 2004;11:345-56.

8. Hoftijzer HC, Bax JJ, Heemstra KA, Bleeker GB, Delgado $\mathrm{V}$, van der Klaauw AA, et al. Short-term overt hypothyroidism induces discrete diastolic dysfunction in patients treated for differentiated thyroid carcinoma. Eur J Clin Invest 2009;39: 204-10.

9. Luster M, Lippi F, Jarzab B, Perros P, Lassmann M, Reiners $\mathrm{C}$, et al. rhTSH-aided radioiodine ablation and treatment of differentiated thyroid carcinoma: a comprehensive review. Endocr Relat Cancer 2005;12:49-64.

10. Hataya Y, Akamizu T, Kanamoto N, Moriyama K, Shimatsu A, Nakao K. A case of subclinical hypothyroidism developing marked pleural effusions and peripheral edema with elevated vascular endothelial growth factor. Endocr J 2007;54:577-84.

11. Parving HH, Hansen JM, Nielsen SL, Rossing N, Munck O, Lassen NA. Mechanisms of edema formation in myxedema-increased protein extravasation and relatively slow lymphatic drainage. N Engl J Med 1979;301:460-5.

12. Kang S, Lee SW, Ahn BC, Lee J. Rapid progression and death in patients with differentiated thyroid cancer after radioiodine 
therapy: report of 4 cases. J Korean Thyroid Assoc 2009;2: 53-61. Korean.

13. Schlumberger M, Catargi B, Borget I, Deandreis D, Zerdoud $\mathrm{S}$, Bridji B, et al. Strategies of radioiodine ablation in patients with low-risk thyroid cancer. N Engl J Med 2012;366:1663-73.

14. Klubo-Gwiezdzinska J, Burman KD, Van Nostrand D, Mete M, Jonklaas J, Wartofsky L. Radioiodine treatment of metastatic thyroid cancer: relative efficacy and side effect profile of preparation by thyroid hormone withdrawal versus recombinant human thyrotropin. Thyroid 2012;22:310-7.

15. Hugo J, Robenshtok E, Grewal R, Larson S, Tuttle RM. Recombinant human thyroid stimulating hormone-assisted radioactive iodine remnant ablation in thyroid cancer patients at intermediate to high risk of recurrence. Thyroid 2012;22: 1007-15. 